\title{
Les stratégies énergétiques régionales en Basse- Bavière face aux enjeux économiques, environnementaux et sociaux
}

Regional energy strategies in the conflict of economical, environmental and social interests on the example of Lower Bavaria

Regionale Energiestrategien im Spannungsfeld ökonomischer, ökologischer und sozialer Interessen am Beispiel Niederbayern

Roland Zink

\section{OpenEdition}

\section{Journals}

Electronic version

URL: http://journals.openedition.org/rge/5478

ISSN: $2108-6478$

Publisher

Association des géographes de l'Est

Printed version

Date of publication: 1 January 2015

ISSN: 0035-3213

\section{Electronic reference}

Roland Zink, «Les stratégies énergétiques régionales en Basse-Bavière face aux enjeux économiques, environnementaux et sociaux », Revue Géographique de l'Est [Online], vol. 55 / n¹-2 | 2015, Online since 09 June 2015, connection on 08 September 2020. URL : http://journals.openedition.org/rge/5478

This text was automatically generated on 8 September 2020

Tous droits réservés 


\title{
Les stratégies énergétiques
} régionales en Basse-Bavière face aux enjeux économiques, environnementaux et sociaux

\author{
Regional energy strategies in the conflict of economical, environmental and \\ social interests on the example of Lower Bavaria \\ Regionale Energiestrategien im Spannungsfeld ökonomischer, ökologischer und \\ sozialer Interessen am Beispiel Niederbayern
}

Roland Zink

\section{Introduction}

1 Les objectifs politiques ambitieux de la transition énergétique en Allemagne font que les municipalités, les Kreis et les régions sont confrontés à de nouveaux défis car ils ont l'obligation d'effectuer la transformation du secteur énergétique de manière durable. Ce sont surtout les territoires ruraux qui sont concernés, en raison de leur grande disponibilité en terres pour les énergies renouvelables. On reconnait de plus en plus le potentiel de développement de ces technologies et on s'efforce de créer sur place une nouvelle valeur ajoutée. Cependant, l'absence de coordination pour l'implantation de ces énergies, ainsi que leur orientation première en fonction des subventions publiques conduisent souvent à une mauvaise répartition spatiale et à des frictions sociales. En fonction des obligations relevant de cette tâche à la fois locale et régionale, cet article évalue les chances de réaliser une transition énergétique qui soit non seulement supportable pour l'environnement, mais aussi économiquement et socialement responsable.

2 Avec la décision prise de transformer son système énergétique, l'Allemagne joue dans le monde un rôle de précurseur sur la voie de l'approvisionnement énergétique 
durable. Le développement des énergies renouvelables constitue une tâche essentielle avec les économies d'énergie et l'amélioration de l'efficacité énergétique. Depuis l'entrée en vigueur du soutien financier par l'État (loi EEG) en 2000, les grandes éoliennes, le photovoltaïque sur toiture et sous forme de grands parcs, ainsi que les installations de biogaz marquent de plus en plus les paysages. Ils sont ainsi le signe visible de la transition énergétique et sont en même temps la manifestation de la voie suivie.

D'une part, les communes et les villes peuvent bénéficier de ce développement, dans la mesure où des activités économiques modernes se créent sur place. D'autre part, avec la mise en œuvre de la transition énergétique, les administrations municipales et leurs dirigeants sont confrontés à des tâches entièrement nouvelles. Par conséquent, le débat sur le futur concept d'approvisionnement énergétique est plus que jamais d'actualité ; mais les questions concernant la localisation des énergies renouvelables et leur intégration dans les structures d'approvisionnement existantes, de manière économiquement viable, écologiquement et socialement responsable, restent encore largement sans réponses.

Photo 1 : parc photovoltaïque en Basse Bavière

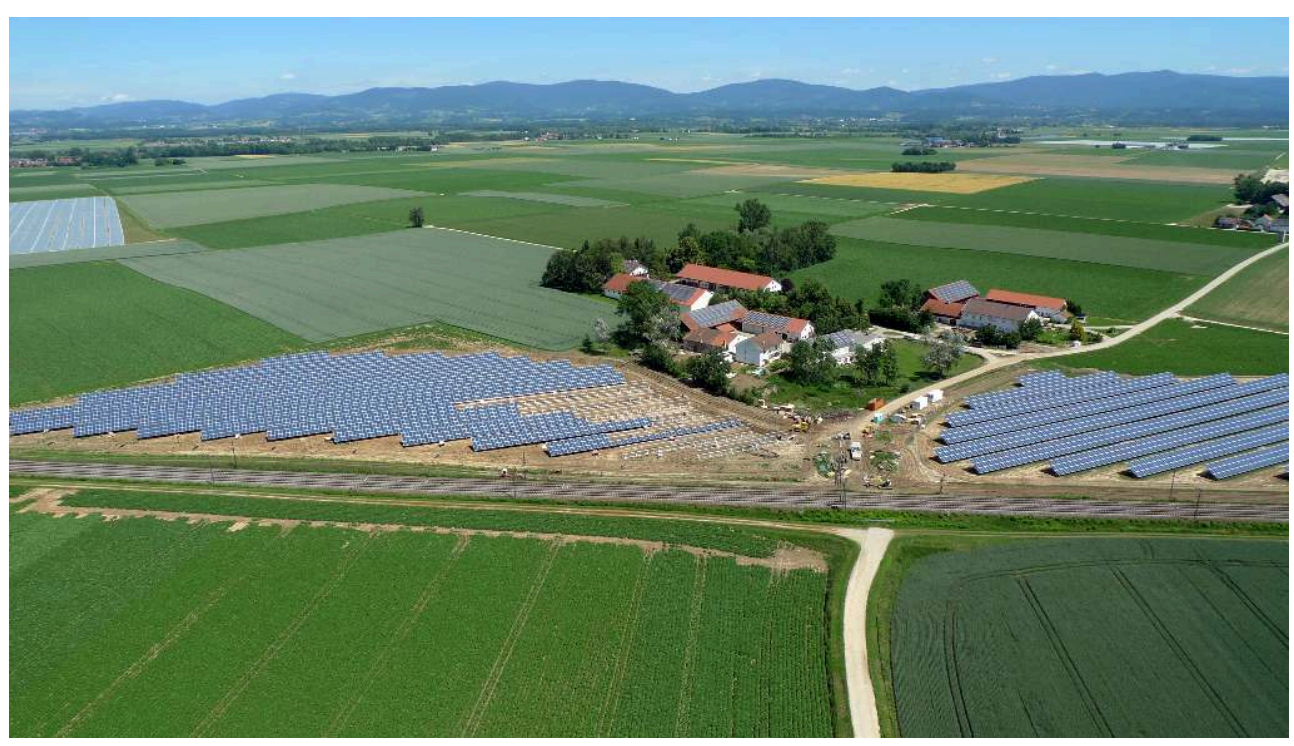

\section{Les enjeux des stratégies régionales}

\section{A. Les enjeux communaux}

L'aménagement durable du territoire a une importance essentielle pour la transition énergétique car l'utilisation accrue des énergies renouvelables conduit à de nouvelles exigences d'espace et à de nouveaux modèles de localisation des systèmes d'approvisionnement énergétique. Ainsi, les systèmes centralisés à forte intensité capitalistique que constituent les grandes centrales sont remplacées par de petites structures dispersées dans l'espace (voir Brücher 2008 et 2009). En raison du fort impact spatial des énergies photovoltaïque, hydraulique et éolienne, ainsi que de la biomasse, il existe de nombreuses zones de conflits locaux. Mentionnons en particulier l'influence croissante sur le paysage, ainsi que sur le cadre de vie de beaucoup de gens ; 
ce qui conduit souvent localement à la formation de résistances et à des préoccupations de conservation de la nature (voir Bosch et Peyke 2010; Zink et al. 2012).

Il apparait de plus en plus souhaitable, en particulier dans les zones rurales, de pouvoir disposer d'une source d'énergie autonome, dans l'espoir de renforcer la structure économique régionale et de donner une impulsion au développement régional durable (voir Tischer et al. 2006; Born 2011; Zink 2013). Au cours des dernières années cependant, il est apparu que les municipalités réagissaient très différemment face à ces nouvelles tâches. Certaines sont par exemple très favorables aux fermes solaires ou éoliennes et font preuve d'un véritable volontarisme, alors que dans d'autres municipalités, la population et les décideurs rejettent majoritairement ces technologies. Des stratégies énergétiques régionales peuvent aider à rapprocher les différents points de vue et les actions contradictoires dans le domaine de l'énergie, afin de construire des structures spatiales pertinentes et durables. La qualité de ces stratégies se mesure de plus en plus à la manière dont elle réussit à s'adapter aux particularités régionales tout en tenant compte des divers enjeux économiques, environnementaux et sociaux.

\section{B. Les enjeux économiques, environnementaux et sociaux}

6 Au moins depuis l'agenda 21, la durabilité est devenue le principe directeur s'appliquant aussi bien à de nombreux domaines du développement mondial que du développement régional ou local. Pour la mise en œuvre des stratégies de l'Agenda 21, de nombreuses municipalités et villes ont déjà acquis une expérience précieuse dans divers domaines et ont pu ainsi contribuer à améliorer la qualité de vie dans leur région. Bien que le concept de durabilité soit utilisé aujourd'hui de manière inflationniste, on reconnaît que le développement durable nécessite la connexion entre les dimensions économiques, écologiques et sociales. En ce qui concerne le secteur de l'énergie, cette attention holistique exige dans tous les domaines de la vie une nouvelle prise de conscience dans le traitement de l'énergie (cf. Hausladen \& Hamacher, 2011). Cela est particulièrement vrai pour le développement des énergies renouvelables et la planification durable de l'emplacement des installations.

7 Pour être conformes aux trois dimensions du développement durable, les nouvelles installations doivent fournir de l'énergie au moindre coût possible et en même temps être économiquement rentables, afin qu'il existe pour les investisseurs une véritable incitation à leur construction et à leur exploitation. De même, les aspects écologiques doivent être pris en compte afin que la compatibilité avec la nature et l'environnement soit assurée. Au cours des dernières années, on a aussi accordé de plus en plus de valeur à l'aspect social. Il arrive ainsi que l'installation d'infrastructures nécessaires au secteur de l'énergie ne puisse être réalisée à cause de problèmes d'acceptation résultant souvent de procédures de planification non transparentes ou de l'absence de participation des citoyens. Cela est particulièrement vrai pour les systèmes d'énergie renouvelable, en raison de leur disposition décentralisée au plus près des gens; ce qui fait qu'elles peuvent grandement influer sur l'environnement d'un site (par exemple les éoliennes).

8 Il semble indispensable de mettre en place des procédures de planification transparentes, ainsi que la possibilité de participer réellement aussi bien à la stratégie de développement qu'à sa mise en œuvre. C'est seulement de cette manière que l'on 
peut aboutir à des solutions socialement acceptables et par conséquent durables. L'avenir de l'approvisionnement énergétique doit donc se conformer et se mesurer à ces différents critères de durabilité (voir SRU 2011). Le rapport des différentes dimensions les unes par rapport aux autres reste cependant contradictoire. Ainsi, même si la prise en compte à égalité de niveau, des différents objectifs est souvent revendiquée, dans les faits on constate que " les objectifs de sécurité d'approvisionnement, de lutte contre le changement climatique et de compétitivité ne peuvent pas être également satisfaits parce qu'ils ont des effets conflictuels » (Kemfert et Müller, 2007, pp 14f). Bien que les dimensions de durabilité soient réduites à certains aspects, il y a de grandes difficultés à satisfaire en même temps toutes les exigences. Il reste à définir, d'une part des critères de développement durable dans le secteur de l'énergie et d'autre part à identifier les façons dont peut les mettre en œuvre.

\section{L'approvisionnement énergétique durable : du concept à la mise en oeuvre}

\section{A. Le concept de double durabilité}

La réalisation d'un approvisionnement énergétique régional durable est une tâche stratégique qui doit être basée sur un plan d'action prédéterminé. Cependant, la durabilité ne possède pas une définition unique car elle s'applique à différents thèmes et elle fait l'objet de diverses approches théoriques ; si bien qu'il faut l'adapter au secteur de l'énergie. Krewitt et al. (2007, p.27), qualifient un approvisionnement énergétique de durable " s'il est basé sur une disponibilité suffisante et durable de ressources énergétiques appropriées et si dans le même temps les effets négatifs de l'approvisionnement et du transport de l'énergie" sont limités. Deux questions principales sont à discuter; d'une part, le choix de la technologie appropriée et d'autre part son utilisation durable sur l'ensemble de la chaîne de processus.

D'un point de vue spatial, il semble également nécessaire d'élargir la définition de Krewitt et al. (2007) en prenant en considération la région comme lieu d'application pour la conception et la mise en œuvre de la durabilité dans le secteur de l'énergie. Un approvisionnement énergétique durable consiste donc, à long terme, à couvrir les besoins énergétiques d'une région avec des technologies durables, assurant un approvisionnement économiquement rentable, tout en étant socialement et écologiquement responsable (voir Zink 2012). L'approche ainsi adoptée se compose de deux parties allant de la sélection à l'utilisation de la technologie ; ce qui d'un point de vue spatial correspond au concept de double durabilité. 
Figure 1 : le concept de double durabilité (Zink)

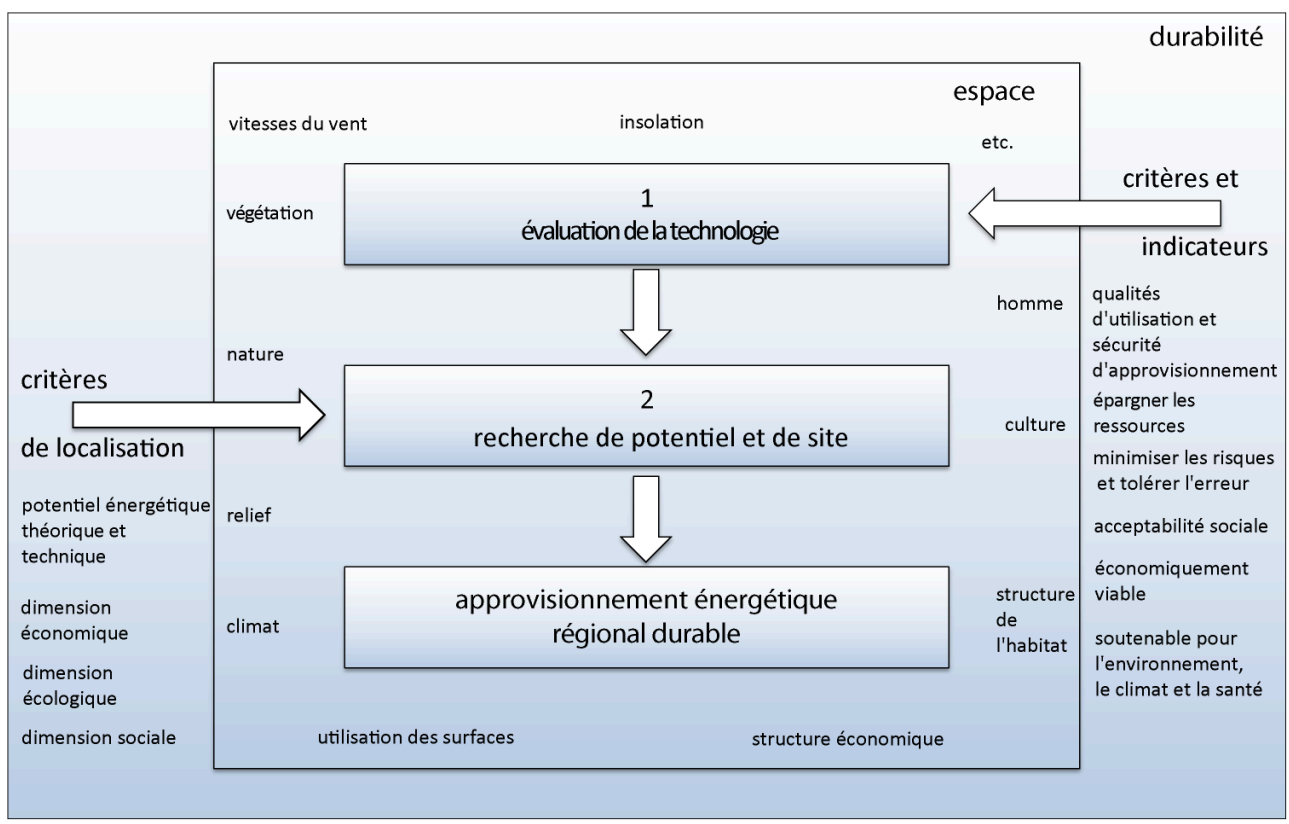

11 Les nécessités du modèle résultent d'une part du choix des technologies, d'autre part de la nécessité de les utiliser dans la région sur une base économique viable, tout en étant socialement soutenable et en respectant l'environnement. Cette double référence à la durabilité reflète la structure de la construction du concept qui s'appuie sur les deux domaines de l'évaluation de la technologie et de son implantation spatiale (voir figure 1). Le concept de durabilité est le concept général de référence. Il définit avec ses principes, ses normes minimales, ses critères et ses indicateurs, les propriétés que doit satisfaire un approvisionnement énergétique durable. Ces exigences s'appliquent aussi bien à l'évaluation de la technologie qu'au choix d'un emplacement approprié. Quand on sait quelles caractéristiques (critères et indicateurs) doivent être remplies pour le développement durable, ce qui va compter en définitive c'est la distribution spatiale prédominante; autrement dit quelles technologies vont s'avérer pertinentes pour chaque région et comment elles vont pouvoir contribuer par un choix judicieux de l'emplacement, à un approvisionnement énergétique qui soit à la fois économiquement viable, écologiquement et socialement responsable.

\section{B. L'évaluation technologique régionale}

La première étape de l'évaluation des technologies est essentielle, car non seulement les potentialités énergétiques régionales sont inégales, mais aussi le modèle de l'approvisionnement énergétique durable, ainsi que ses préférences, résultent d'un discours sociétal. C'est pourquoi, on peut en fonction des spécificités régionales, privilégier des options technologiques très différentes (Hake et Eich 2005, p 11). Pour chaque portion d'espace considéré, il faut donc sélectionner les technologies qui, au regard des conditions locales et globales, peuvent fournir un approvisionnement énergétique optimum et assurer la meilleure contribution à la réalisation de la durabilité (Heimann 2001, p 14). Dans ce cas, aucune technologie ne peut remplir aussi bien tous les critères et les objectifs de la durabilité (SRU 2011, p 15), puisque chaque technologie présente des forces et des faiblesses spécifiques. L'évaluation permet de 
montrer quelles technologies répondent le mieux aux exigences du développement durable dans un contexte spatial donné.

\section{La recherche de potentiels et de sites d'implantation}

13 Les technologies énergétiques durables identifiées dans une première étape doivent ensuite être appliquées pour l'approvisionnement énergétique dans la zone d'étude, avec si possible la nécessité d'éviter des effets négatifs sur l'environnement et les populations. Une planification habile et adaptée aux critères de la durabilité peut apporter une contribution précieuse. Cette approche prend en compte le fait qu'un système composé de technologies énergétiques durables n'est pas nécessairement en conformité avec le modèle du développement durable (par exemple, la nécessité de déplacer et de réinstaller des personnes en raison de la construction d'une centrale hydroélectrique). Par conséquent, l'objectif doit être l'aménagement durable du territoire, qui consiste à intégrer dans l'espace considéré les technologies énergétiques appropriées de façon économiquement rentable, socialement acceptable et dans le respect de l'environnement. En conséquence de cette exigence, il semble nécessaire de mener une approche qui accorde une place centrale aux trois dimensions de la durabilité et prépare les lignes directrices pour l'analyse des sites d'implantation.

\section{L'offre régionale d'énergie durable}

e choix des technologies énergétiques appropriées ainsi que leur modélisation dans le contexte régional se font étape par étape avec chacune sa propre référence à la durabilité qui est à construire progressivement. Ensemble, ils forment le noyau central de la double durabilité et donnent la stratégie à suivre pour atteindre un approvisionnement énergétique durable. L'espace d'étude réunit les composantes les reliant les uns aux autres. Grâce à lui, les exigences de la durabilité (critères, indicateurs et caractéristiques de localisation) sont remplies, si bien que le système énergétique présente un caractère particulier en relation avec l'espace (voir Brücher 2009). Ce sont non seulement les formes d'énergie, mais aussi le modèle de répartition des installations qui sont tributaires des caractéristiques naturelles, socio-économiques et socio-culturelles de l'espace, ainsi que des valeurs et des modes de vie des personnes vivant à l'intérieur de la zone d'étude. Par conséquent, la participation des citoyens, des acteurs du secteur de l'énergie, des décideurs et des autres parties intéressées aux procédures de stratégie et de planification est d'une grande importance.

15 En ce qui concerne l'évaluation de la technologie ainsi que la recherche d'un site, le concept consiste à permettre aux groupes mentionnés ci-dessus, de définir eux-mêmes les critères et leurs préférences de localisation et donc ainsi de concevoir la forme d'approvisionnement énergétique. Avec cette approche, on peut réussir à faire participer les citoyens ; ce qui leur donne une grande estime de soi et en même temps contribue à l'acceptation croissante des mesures prises. En fonction de ces divers facteurs ayant une influence spatiale, l'application du concept proposé sur différents espaces de recherche peut donc conduire à diverses formes d'approvisionnement énergétique durable. 


\section{Stratégie régionale de l'énergie : l'exemple de la Basse-Bavière}

Au cours des dernières années, le Bezirk de Basse-Bavière s'est à plusieurs reprises beaucoup investi dans le développement des énergies renouvelables, en particulier le photovoltaïque (voir Zink 2013). Ainsi, le premier parc photovoltaïque de plein champ a été construit à Sonnen (Landkreis Passau) en 2002. Il a entre autres été suivi par le premier parc solaire d'Allemagne de $10 \mathrm{MWc}$ sur une zone de reconversion militaire à Pocking (Landkreis Passau), ainsi que par ce qui était en 2009 le deuxième plus grand parc solaire au monde, à Straßkirchen (district de Straubing -Bogen), avec une puissance de 54 MWc et une surface d'environ 140 hectares. Il faut aussi mentionner le projet d'une usine de pompage-turbinage de $300 \mathrm{MW}$ dans la commune de Riedl (Landkreis Passau), ou la forte expansion annoncée de l'énergie éolienne dans la Forêt de Bavière. Bien que la Basse-Bavière ait entrepris des efforts importants dans le développement des énergies renouvelables (la proportion d'électricité bénéficiant du tarif d'achat de la loi EEG s'élève déjà à $43 \%$; voir DGS 2013, situation en octobre 2013) qui contribuent ainsi de manière significative à la transformation du système énergétique, la voie suivie est controversée (voir Zink 2010).

Lors de l'étape d'évaluation des technologies, l'application du concept de la double durabilité a été en mesure de permettre le développement des énergies renouvelables dans le Regierungsbezirk; mais en même temps, le plan d'implantation a fait ressortir des erreurs et a d'autre part présenté une stratégie d'expansion. En relation avec le concept de durabilité (voir Kopfmüller et al 2000 ; Fischedick et Nitsch 2002), on a pu définir des critères ou des lignes directrices de durabilité qui ont formé le cadre de l'analyse lors de la première étape de l'évaluation de la technologie. L'évaluation se fondait finalement sur une procédure de prise de décision comportant 14 indicateurs qui ont permis une quantification des faits et une comparaison des technologies. Le choix des indicateurs a été fait au moyen d'une évaluation complète des autres ensembles d'indicateurs plutôt que sur l'évaluation des technologies de l'énergie (13 études au total). En outre, les citoyens et les acteurs du secteur de l'énergie pouvaient pondérer les lignes directrices et faire valoir leurs préférences (voir figure 2).

Figure 2 : critères d'évaluation

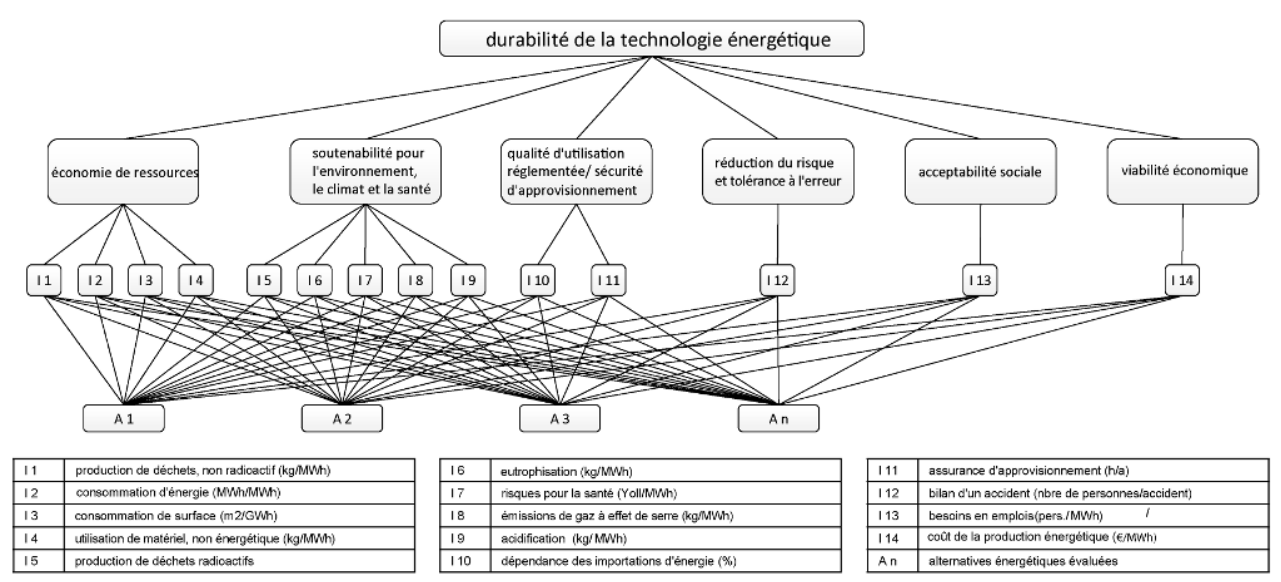



renouvelables sont dans l'ensemble à privilégier. Cela concerne en particulier l'hydroélectricité et l'énergie éolienne, suivie par le photovoltaïque de toiture, la biomasse et les parcs photovoltaïques. Dans le même temps, les centrales thermiques et nucléaires occupent les dernières places. Ce classement est en conformité avec une enquête sur l'acceptabilité menée auprès des citoyens et des acteurs, pour qui toutes les énergies renouvelables doivent à l'avenir être considérablement développées dans leur région, alors qu'il faut renoncer au nucléaire et au charbon et utiliser les centrales à gaz au même degré qu'aujourd'hui. Cette appréciation positive des énergies renouvelables est cependant enregistrée tant que l'éolienne ou l'usine de biogaz ne vient pas perturber leur cadre de vie. Par conséquent, la notion de double durabilité implique qu'après avoir vérifié l'aptitude de la technologie, il faut l'implanter dans la région de manière durable.

19 La modélisation des sites d'implantation potentiellement durables se fait à l'aide d'un système d'information géographique (SIG), ainsi que d'un catalogue qui comporte les critères d'exclusion et de conformité sur la base des dimensions de la durabilité. La référence à la durabilité est abordée suivant une analyse de potentiels (voir Kaltschmitt et al. 2006) faite avec les trois dimensions de l'économie, de l'écologie et de la société (voir commission d'enquête 1998). L'égale attention accordée aux différentes dimensions peut conduire à l'exclusion d'un site, aussi bien à cause de l'aspect écologique que pour des raisons économiques ou sociales. Les critères de sélection du site sont d'abord le potentiel technique de l'énergie, qui est défini par les conditions géologiques ou naturelles du site tels que le rayonnement solaire, les vitesses moyennes du vent, ou les conditions de croissance de la biomasse végétale. La possibilité d'utiliser une technologie pour une région donnée varie en fonction des différents usages potentiels et des caractéristiques socio-économiques ou socio-culturelles de l'espace (voir Porsche 2010 et figure 3). 
Figure 3: représentation cartographique des sites d'implantations énergétiques durables en BasseBavière à l'aide de SIG (Source: Zink)

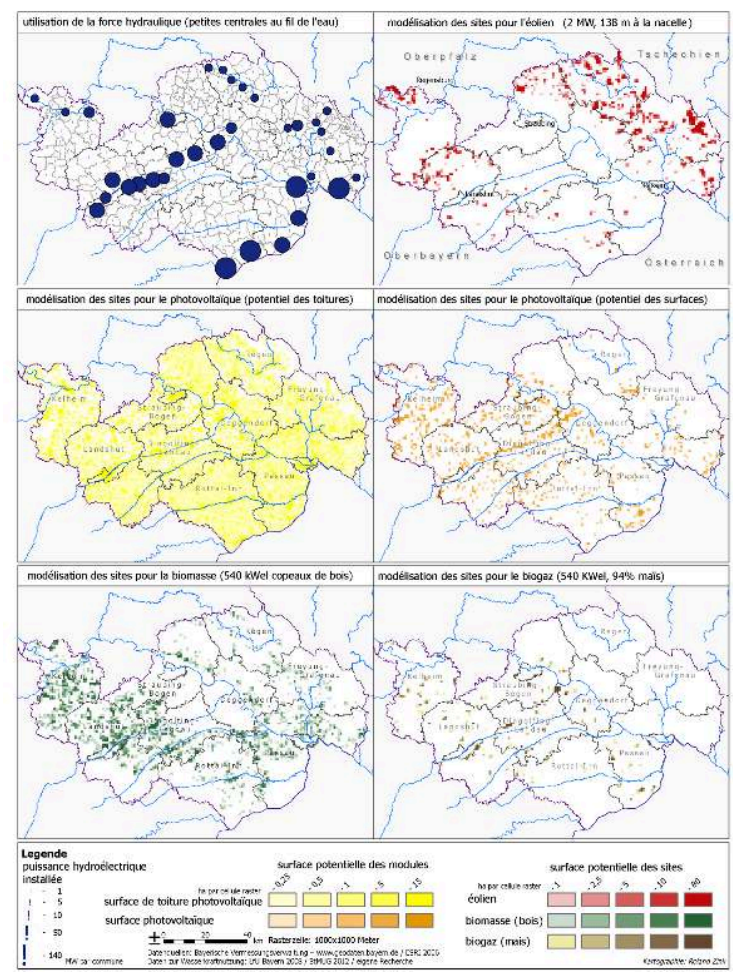

Il existe différents schémas de localisation en fonction des conditions à remplir pour pouvoir utiliser de manière durable les énergies renouvelables. La modélisation du site d'implantation a été effectuée après avoir procédé à l'évaluation des technologies. En ce qui concerne l'hydroélectricité, la Basse-Bavière bénéficie des cours d'eau abondants que sont le Danube, l'Inn et l'Isar qui traversent le Regierungsbezirk. Le potentiel hydroélectrique de ces rivières en particulier, ainsi que celui de cours d'eau plus petits sont déjà complètement utilisés aujourd'hui, de sorte qu'à l'exception de mesures de modernisation ou de réactivation il n'existe que peu d'options d'extension. En revanche, l'énergie éolienne ne joue jusqu'ici qu'un rôle mineur. De nombreux sites potentiels se trouvent dans la Forêt de Bavière, là où il existe un accès à des sites d'implantation, là où les vitesses du vent sont suffisantes, où il n'y a pas de problèmes de protection de la nature et où il existe une distance suffisante par rapport aux zones habitées.

Tout comme l'hydroélectricité, l'énergie photovoltaïque est déjà pleinement utilisée. Le potentiel sur les toitures suit la structure de l'habitat et montre une distribution extrêmement décentralisée. Les emplacements possibles pour les systèmes au sol sont principalement situés le long des grandes infrastructures de transport, ainsi que sur des surfaces précédemment occupées (friches industrielle ou commerciales et surfaces à réhabiliter). Il est surprenant que les sites d'implantation pour les centrales à biogaz ainsi que pour les unités de cogénération (à copeaux de bois) se limitent aux collines des dépôts tertiaires et au piémont de la Forêt de Bavière. Cela est dû à la taille des installations. Dans cet espace il y a suffisamment de matière première pour le fonctionnement des centrales et en même temps il y a assez de gros consommateurs d'énergie pour permettre la production combinée de chaleur et d'électricité. 


\section{Conclusion}

La transformation du système énergétique polarise l'attention sur les zones rurales comme le Regierungsbezirk de Basse-Bavière. Mais élargir l'importance des énergies renouvelables n'est pas suffisant pour la transition énergétique souhaitée. D'une part, les énergies renouvelables ne respectent pas dans toutes les régions les critères de durabilité et d'autre part, le simple appel à plus de renouvelables ne suffit pas à une mise en œuvre concrète, ou à l'intégration dans des structures d'approvisionnement. Le concept de la double durabilité doit montrer comment concevoir un approvisionnement énergétique régional conforme aux principes du développement durable. Pour cela, l'observation spatiale intégrée joue un rôle important. Elle peut apporter une contribution significative à l'aménagement et à la planification durable des systèmes énergétiques et permettre une combinaison de l'évaluation des technologies des choix de sites.

Le modèle de la durabilité fournit la direction à suivre pour les deux secteurs. Cependant, il doit être d'abord intégré dans le développement d'une stratégie énergétique avant d'être opérationnel pour l'application pratique. Le concept présenté utilise d'une part la notion de développement durable et d'autre part le modèle des trois piliers. Dans le respect des critères de durabilité ainsi obtenus, les infrastructures municipales et régionales d'approvisionnement énergétique peuvent être efficacement modélisées en utilisant les SIG. Les résultats montrent comment le futur approvisionnement en énergie peut ou doit changer, afin de maitriser efficacement les grands défis du secteur de l'énergie.

\section{BIBLIOGRAPHY}

Brücher W., 2008, « Erneuerbare Energien in der globalen Versorgung aus historischgeographischer Perspektive », Geographische Rundschau, 1, p.4-12.

Brücher W., 2009, Energiegeographie - Wechselwirkungen zwischen Ressourcen, Raum und Politik, Stuttgart.

Born K.-M., 2011, "Ländliche Räume in Deutschland, Differenzierungen, Entwicklungspfade und brüche”, Geographische Rundschau, 2, p.4-10.

Bosch S. \& Peyke G., 2010, "Raum und Erneuerbare Energien, Anforderungen eines regenerativen Energiesystems an die Standortplanung”, Zeitschrift für angewandte Geographie, 1, p.11-19.

Deutsche Gesellschaft für Sonnenenergie e.V. (DGS), 2013, Datenbank EnergyMap.info zur EEGVergütung in Niederbayern, http://www.energymap.info/energieregionen/DE/ 105/111/165.html, Zugriff 10/10/2013.

Enquete-Kommission des 13. Deutschen Bundestages, 1998, Konzept Nachhaltigkeit, vom Leitbild zur Umsetzung, Bonn. 
Fischedick M. \& Nitsch J., 2002, Langfristszenarien für eine nachhaltige Energienutzung in Deutschland, Berlin.

Hake J.-F. \& Eich R., 2005, “Anforderungen an eine in die Zukunft gerichtete Energieforschung”, Energiewirtschaftliche Tagesfragen, 1/ 2, p.8-11.

Hausladen G. \& Hamacher T., 2011, “Leitfaden Energienutzungsplan”, in Bayerisches Staatsministerium für Umwelt und Gesundheit (StMUG), Bayerisches Staatsministerium für Wirtschaft, Infrastruktur, Verkehr und Technologie (StMWIVT) \& Oberste Baubehörde im Bayerischen Staatsministerium des Inneren (OBB) (dir), München, http://www.stmi.bayern.de/ imperia/md/content/stmi/bauen/rechtundtechnikundbauplanung/_staedtebau/ veroeffentlichungen/oeko/leitfaden_energienutzungspl_1.pdf, Zugriff 22/03/2011.

Heimann M., 2001, Methodik zur technischökologischen Bewertung regenerativer Energiequellen zur Erzeugung elektrischer Energie, Bochum.

Kaltschmitt M., Streicher W. \& Wiese A. (dir.), 2006, Erneuerbare Energien, Systemtechnik, Wirtschaftlichkeit, Umweltaspekte, Berlin, Heidelberg.

Kopfmüller J., Coenen R., Jörissen J., Fleischer T., Rösch C., Sardermann G., Schulz V., Langniß O. \& Nitsch J., 2000, Konkretisierung und Operationalisierung des Leitbildes einer nachhaltigen Entwicklung für den Energiebereich, http://www.itas.fzk.de/deu/Itaslit/koua00a.pdf, Zugriff 17/03/2011.

Krewitt W., Nitsch J., Langniß O. \& Fischedick M., 2007, “Leitlinien für eine nachhaltige Energieversorgung", in Forschungsverbund Sonnenenergie (dir.), Forschung und Innovation für eine nachhaltige Energieversorgung, Themen 2006, Berlin, p.27-31.

Porsche L., 2010, "Stadtentwicklung voller erneuerbarer Energie”, Informationen zur Raumentwicklung, 9, p.665-673.

Sachverständigenrat für Umweltfragen (SRU) (dir.), 2011, Wege zur 100\% erneuerbaren Stromversorgung, Sondergutachten, http://www.umweltrat.de/SharedDocs/Downloads/DE/ 02_Sondergutachten/2011_Sondergutachten_100Prozent_Erneuerbare.pdf? blob=publicationFile, Zugriff 21/03/2011.

Tischer M., Stöhr M., Lurz .M \& Karg L., 2006, Auf dem Weg zur 100\% Region, Handbuch für eine nachhaltige Energieversorgung von Regionen, München.

Zink R., 2010, "Einbindung von GIS in einen nachhaltigen Planungsprozess von Photovoltaik Freiflächenanlagen auf kommunaler Ebene", in Strobl J., Blaschke T. \& Griesebner G. (dir.), Angewandte Geoinformatik 2010, Beiträge zum 22. AGIT-Symposium Salzburg, p.954-963.

Zink R., 2012, Raum für Energie. Ein integratives Konzept zur Modellierung einer regionalen nachhaltigen Energieversorgung, Dissertation an der Universität Passau.

Zink R., 2013, "Ländliche Entwicklung durch erneuerbare Energien - Das Beispiel Photovoltaik in Niederbayern", Standort, 37, H. 3, p.172-178.

Zink R., Diepold F. \& Pagany R. (2012), 3D-Visualisierung am Beispiel der Windenergie, GIS.BUSINESS, H. 3, p.32-35.

\section{ABSTRACTS}

Ambitious statements on national energy policy provide new challenges for municipalities, counties and regions, because they are responsible for the design and implementation of the 
energy sector's sustainable transformation. Especially rural areas realize the potential of renewable energies as a development opportunity and aspire to generate local added value. As a consequence of the primary focus on economic profitability or state subsidies, the absence of a comprehensive coordinated site planning often leads to spatial misallocation and social frictions. This paper identifies the potential of a profitable, ecological, socially acceptable and sustainable energy system. Moreover a new guideline for the design of a regional sustainable energy system and its application to the District of Lower Bavaria is presented.

Les objectifs ambitieux de la politique énergétique nationale constituent de nouveaux défis pour les municipalités, les Kreis et les régions qui sont responsables de la conception et de la mise en œuvre de la transformation durable du secteur de l'énergie. Les zones rurales en particulier ont pris la mesure du potentiel des énergies renouvelables comme opportunité de développement et aspirent à générer de la valeur ajoutée locale. Avec l'attention accordée à la rentabilité économique ou aux subventions publiques, l'absence d'un plan complet et coordonné d'implantation conduit souvent à une mauvaise répartition spatiale et à des frictions sociales. Cet article présente le potentiel d'un système énergétique rentable, écologique, socialement acceptable et durable. En outre, on présente un nouveau cadre directeur pour la réalisation d'un système énergétique régional durable à travers une application dans le district de Basse-Bavière.

Die hochgesteckten politischen Absichtserklärungen zur nationalen Energiewende in Deutschland stellen Kommunen, Landkreise und Regionen vor neue Herausforderungen, da es ihnen obliegt, die Transformation am Energiesektor nachhaltig zu gestalten. Gerade ländlich geprägte Gebiete rücken dabei aufgrund ihrer hohen Flächenverfügbarkeit für erneuerbare Energien in den Fokus. Dort erkennt man zunehmend das Entwicklungspotenzial dieser Technologien und ist bestrebt, neue Wertschöpfung vor Ort zu generieren. Das Fehlen einer koordinierten Standortplanung führt allerdings ebenso wie die primäre Orientierung an staatlicher Subventionierung vielfach zu räumlichen Fehlallokationen und gesellschaftlichen Friktionen. Entsprechend dieser kommunal-regionalen Aufgabe setzt sich dieser Beitrag mit den Chancen einer ökonomischen, ökologischen und sozialverträglichen Gestaltung der Energiewende in Niederbayern auseinander.

\section{INDEX}

Schlüsselwörter: doppelte Nachhaltigkeit, ländlich geprägte Gebiete, Niederbayern, regionale Energiestrategie

Mots-clés: Basse Bavière, double durabilité, espaces ruraux, stratégie régionale de l'énergie

Keywords: double sustainability, low Bavaria, regional energy strategy, rural areas

\section{AUTHOR}

\section{ROLAND ZINK}

Universität Passau - roland.zink@uni-passau.de 